\section{Mit Aromatherapie Hormone beeinflussen}

Tarumi W, Shinohara K. The effects of essential oil on salivary oxytocin concentration in postmenopausal women. J Altern Complement Med 2020; 26: 226-230. doi:10.1089/acm.2019.0361

Japanische Forscher untersuchten bei je 15 postmenopausalen Frauen den Einfluss verschiedener Aromen im Cross-over-Design auf Oxytocin-Spiegel im Speichel im Vergleich zu einer Kontrolle. In der Einleitung beziehen sich die Autoren auf 2 eigene Arbeiten über den entsprechenden Einfluss von Aromen auf Estrogen bzw. Testosteron im Speichel. Postmenopausaler Muskelabbau geht mit niedrigen Oxytocin-Spiegeln einher und würde durch eine Erhöhung der Spiegel gehemmt.

Die Auswahl von je 15 nicht rauchenden Frauen für jedes der 10 untersuchten ätherischen Öle erfolgte über eine seit mindestens 12 Monaten bestehenden Amenorrhoe. Die Probandinnen gaben ihr Einverständnis und es lag angeblich ein Ethikvotum vor. Bezüglich der Fallzahl wird einfach auf die beiden früheren Studien Bezug genommen.

\section{Zehn ätherische Öle im Test}

Die untersuchten 10 ätherischen Öle wurden in einem Vorversuch mit 7 prämenopausalen Frauen so verdünnt, dass es von keiner unangenehm beurteilt wurde. Dipropylenglykol wurde als Verdünnungsmittel und als Kontrolle verwendet, bei Rose Otto (Rosenöl) und Sandelholz wurde laut Tab. 2 der Arbeit Propylenglykol zur Verdünnung eingesetzt. Unverdünnt blieb lediglich Süßorange. Die finalen Konzentrationen lagen nach Tab. 2 zwischen $0,75 \%$ und $100 \%$ : Rose Otto (6\%), Süßorange (100\%), Lavendel (30\%), Neroli (7,5\%), Weihrauch (45\%), Jasmin absolute (12\%), Ylang-Ylang (1,5\%), Römische Kamille (0,75\%), Muskatellersalbei (12\%) und Indisches Sandelholz (30\%).

In Tab. 2 werden ferner die Hauptkomponenten der ätherischen Öle quantifiziert genannt, woher diese Angaben stammen, ist unklar. Da der genannte Lieferant ein Chemikalienhändler ist, bleiben Fragen zur Qualität offen.

Die ätherischen Öle wurden über eine genau beschriebene Einrichtung mit einem dosierten Flow über einen Glastrichter in den Nasenbereich gebracht. Die Teilnehmerinnen erhielten jeweils eine 20-minütige Inhalation mit der Kontrolle, danach ebenso lang mit einer der Testsubstanzen. Mit dem Hinweis, dass jede Probandin mit jeweils nur einer Substanz pro Tag getestet wurde, ergibt sich die Möglichkeit einer weiteren Testung an einem Folgetag bei derselben Person. Speichelproben wurden 4-mal pro Sitzung gewonnen: vor und nach Kontrolle sowie vor und nach Testsubstanz. Die Oxytocin-Konzentration im Speichel wurde mit einem kompetitiven ELISA-Kit bestimmt. Aus dem Verhältnis nachher/ vorher wird die „rate of change“ berechnet und in Tab. 3 dargestellt ( Tab. 1).

\section{Ergebnisse}

Alle Öle außer Weihrauch zeigen einen über der Kontrolle liegenden Anstieg. Bei Rose, Süßorange und bei Ylang-Ylang ergibt sich jedoch kein statistisch signifikanter Unterschied, bei letzterem aufgrund einer sehr hohen Streuung. Bei Lavendel, Neroli, Jasmin absolute, Römischer Kamille, Muskatellersalbei ( $\mathbf{A} \mathbf{A b} \mathbf{b} \mathbf{1}$ ) und Sandelholz ist der Anstieg signifikant verschieden versus Kontrolle.

In der Diskussion wird auf die Übereinstimmung bezüglich Oxytocin-Anregung durch Salbei bei schwangeren Frauen hingewiesen. Weiterhin wird die angstlösende Wirkung von Linalool mit der OxytocinErhöhung von Lavendel in Zusammenhang gebracht. Allerdings widerlege das Fehlen einer Oxytocin-Erhöhung unter Linaloolhaltigem Ylang-Ylang eine Erklärung der anxiolytischen Wirkung von Lavendel über nur eine Wirksubstanz - wobei hier weder die breite Streuung noch die sehr starke Verdünnung von Ylang-Ylang in die Überlegungen einfließt.

Tab. 1 (= Tabelle 3 der Originalarbeit) Averages and standard deviations, and $95 \%$ confidence interval (Cl) of salivary oxytocin concentration under each condition.

\begin{tabular}{|l|l|l|l|l|l|}
\hline & Control & $95 \% \mathbf{C l}$ & Essential oils & 95\% Cl & P \\
\hline Rose otto & $1.15 \pm 0.33$ & $0.97-1.34$ & $1.29 \pm 0.33$ & $1.10-1.47$ & 0.2407 \\
\hline Sweet orange & $1.15 \pm 0.29$ & $0.98-1.31$ & $1.35 \pm 0.43$ & $1.11-1.59$ & 0.0877 \\
\hline Lavender & $1.02 \pm 0.45$ & $0.73-1.31$ & $1.62 \pm 0.68$ & $1.19-2.06$ & 0.0011 \\
\hline Neroli & $0.99 \pm 0.33$ & $0.81-1.18$ & $1.33 \pm 0.36$ & $1.13-1.53$ & 0.0066 \\
\hline Frankincense & $1.03 \pm 0.22$ & $0.90-1.16$ & $1.03 \pm 0.21$ & $0.92-1.16$ & 0.9292 \\
\hline Jasmine absolute & $0.99 \pm 0.46$ & $0.73-1.24$ & $1.42 \pm 0.75$ & $1.01-1.84$ & 0.0492 \\
\hline Ylang ylang & $1.10 \pm 0.42$ & $0.86-1.33$ & $1.65 \pm 1.18$ & $0.99-2.30$ & 0.1147 \\
\hline Roman chamomile & $1.01 \pm 0.37$ & $0.80-1.21$ & $1.60 \pm 0.43$ & $1.36-1.84$ & 0.0002 \\
\hline Clary sage & $0.97 \pm 0.28$ & $0.82-1.12$ & $1.43 \pm 0.48$ & $1.16-1.69$ & 0.0031 \\
\hline Indian sandalwood & $1.15 \pm 0.39$ & $0.94-1.37$ & $1.55 \pm 0.61$ & $1.21-1.89$ & 0.0170 \\
\hline
\end{tabular}

The oxytocin concentration is expressed as "rates of change" ([concentration after exposure] / [concentration before exposure]) 


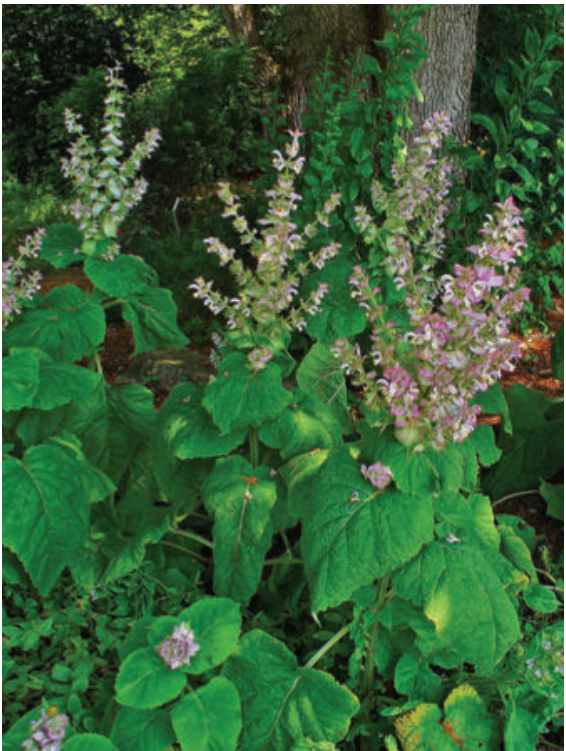

-Abb. 1 Duftet gewürzartig-herb und stimuliert die Oxytocin-Bildung: Muskatellersalbei, hier im Botanischen Garten der Universität Karlsruhe. Quelle: H. Zell

Die Autoren diskutieren, dass weder Blutkonzentrationen von Oxytocin noch klinische Zeichen wie Muskelmasse untersucht wurden, und auch keine Sicherheit. Zukünftige Studien sollten weiter verblindet werden, unterschiedliches Riechvermögen der Probanden berücksichtigen sowie unterschiedliche persönliche Präferenzen. Bei festgelegter Reihenfolge habe die Kontrolle evtl. die Testphasen mit den Aromen beeinflusst. Der Zeitpunkt der Experimente könnte wegen des Tagesrhythmus die Ergebnisse beeinflusst haben. In der Schlussfolgerung meinen die Autoren, dass die olfaktorische Stimulation mit bestimmten ätherischen Ölen trotz der genannten Einschränkungen als eine Anti-Aging-Strategie für postmenopausale Frauen dienen könne.

\section{Kommentar}

Zunächst zur o.g. Schlussfolgerung: Wenn es denn so einfach wäre, dann bräuchten wir ja nur noch das Inhalat für die Männer... Aber im Ernst: Durch die parallele Untersuchung von immerhin 10 ätherischen Ölen jeweils im Vergleich zur Kontrolle - ist diese Studie eine Bereicherung der Aromatherapie-Forschung. Mit dem positiven Ergebnis bei mindestens 6 der Öle wäre eine Diskussion über die Frage zu führen, ob die Oxytocin-Anstiege hier nur recht unspezifisch durch eine olfaktorische Beeinflussung der Stimmung in Richtung „angenehm“ zustande kommen. Leider fehlt hier der Versuch, solche Stimmung für jedes Öl zu erfassen und der Mehraufwand durch ein paar Fragen wäre gering gewesen. Bei der Diskussion über Wirkbeiträge einzelner Komponenten hätte die resultierende Dosierung berücksichtigt werden müssen. Die Autoren weisen selbst darauf hin, dass Dosierungen evtl. individueller anzupassen wären.

Leider hat die Publikation einige Mängel, die den Reviewern oder dem Verlag hätten auffallen müssen: Die Nennung von Komponenten der Testsubstanzen ohne Quelle und ohne Tabellenbeschriftung. Ferner drücken sich die Autoren vor einer Nennung der Probandenzahl insgesamt. Wie leichte
Unterschiede im Mittelwert des Alters bei jeder Testsubstanz zeigen, wurden vermutlich die meisten - aber eben nicht alle Probanden wiederholt getestet. Daraus folgen aber gewisse grundsätzliche Probleme in der Statistik. Die gibt es ohnehin bezüglich des nicht komplett beschriebenen Ausschlusses von 3 Sitzungen. Bei der kleinen Fallzahl dürften die Voraussetzungen für Anwendung des t-Tests für die Gruppenunterschiede nicht vorliegen - aber vermutlich würden verteilungsfreie Tests weitgehend ähnliche Ergebnisse ergeben.

Interessenkonflikt

BU berät Anbieter von meist pflanzlichen Arzneimitteln, Medizinprodukten und Nahrungsergänzungsmitteln - auch solche von traditionellen Arzneimitteln.

\section{Korrespondenzadresse}

Prof. Dr. Dr. Bernhard Uehleke

Charité - Universitätsmedizin Berlin

Abt. für Klinische Naturheilkunde

Immanuel Krankenhaus am Wannsee

Königstr. 63

14109 Berlin

E-Mail: b.uehleke@gmx.net

\section{Bibliografie}

DOI https://doi.org/10.1055/a-1102-1613 Zeitschrift für Phytotherapie 2020; 41: 89-90 (c) Georg Thieme Verlag KG Stuttgart · New York

ISSN 0722-348X 